\title{
New simpler method of matching NLO corrections with parton shower Monte Carlo*
}

\author{
S. Jadach ${ }^{\dagger}$ \\ Institute of Nuclear Physics, Polish Academy of Sciences, \\ ul. Radzikowskiego 152, 31-342 Kraków, Poland \\ E-mail: stanislawejadach@ifj.edu.pl \\ W. Płaczek \\ Marian Smoluchowski Institute of Physics, Jagiellonian University, \\ ul. Łojasiewicza 11, 30-348 Kraków, Poland
}

\section{S. Sapeta}

Theoretical Physics Department, CERN, Geneva, Switzerland

\section{A. Siódmok}

Theoretical Physics Department, CERN, Geneva, Switzerland

\section{Skrzypek}

Institute of Nuclear Physics, Polish Academy of Sciences, ul. Radzikowskiego 152, 31-342 Kraków, Poland

\begin{abstract}
Next steps in development of the KrkNLO method of implementing NLO QCD corrections to hard processes in parton shower Monte Carlo programs are presented. This new method is a simpler alternative to other well-known approaches, such as MC@NLO and POWHEG. The KrkNLO method owns its simplicity to the use of parton distribution functions (PDFs) in a new, so-called Monte Carlo (MC), factorization scheme which was recently fully defined for the first time. Preliminary numerical results for the Higgs-boson production process are also presented.
\end{abstract}

Loops and Legs in Quantum Field Theory

24-29 April 2016

Leipzig, Germany

\footnotetext{
${ }^{*}$ This work is partly supported by the Polish National Science Centre grant UMO-2012/04/M/ST2/00240.

$\dagger$ Speaker.

¥On leave of absence from INP PAS, Kraków, Poland.

${ }^{\S}$ On leave of absence from INP PAS, Kraków, Poland.
} 


\section{Introduction}

The first idea of the of the KrkNLO methodology was suggested at Ustron 2011 Conference [1] as a byproduct of another study on introduction of NLO corrections in the QCD evolution of parton distributions using Monte Carlo (MC) methods. This proposal was originally formulated for the process of $Z / \gamma$ production in proton-proton collisions, i.e. the Drell-Yan (DY) process, and was limited to multi-gluon emission out of quarks (gluonstrahlung). However, numerical implementation was missing. The first numerical validation of the above KrkNLO method was presented shortly in ref. [2] within the so-called "Double-CMC" toy-model parton shower (PS) Monte Carlo (MC), still for gluonstrahlung only.

A more complete theoretical discussion of the KrkNLO scheme, with an explicit introduction of the parton distribution functions (PDFs) in the MC factorization scheme, for DY and electronproton deep-inelastic scattering (DIS) was provided in ref. [3], but the MC implementation was still done on top of the not-so-realistic Double-CMC PS, and for gluonstrahlung in DY process only.

The first realistic implementation of the KrkNLO method was presented in ref. [4]. It was done on top of the Sherpa [5] and Herwig [6, 7, 8] PS MCs, implementing the complete NLO QCD corrections for the DY process, including gluon-quark transitions. In this work the KrkNLO numerical results were compared with these from MC@NLO [9] and POWHEG [10] implementations. Also comparisons with the NLO fixed-order calculations of MCFM [11] and with the NNLO ones of ref. [12] were presented.

In the following we shall elaborate on: (i) a mature definition of PDFs in the MC factorization scheme, (ii) universality of the MC factorization scheme, (iii) application to the Higgs-production process. These issues are also covered in the recent ref. [13].

\section{KrkNLO method}
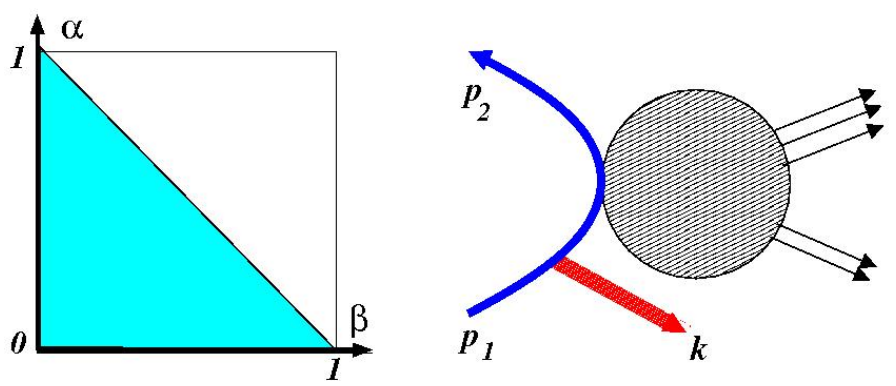

Figure 1: Kinematics of the $g+g \longrightarrow H, H+g$ process.

A recipe of the KrkNLO method of introducing NLO QCD corrections for spin-zero Higgsboson production in proton-proton collisions is unbelievably simple: take an event generated by a LO-type parton shower MC as it is and apply a simple positive well-behaved weight

a) for the subprocess $g+g \longrightarrow H$ :

$$
W_{V S}^{\mathrm{MC}}(\alpha, \beta)=1+\Delta_{V S}^{\mathrm{MC}},
$$


where

$$
\Delta_{V S}^{\mathrm{MC}}=\frac{\alpha_{s}}{2 \pi} 2 C_{A}\left[\frac{473}{72}+\frac{2 \pi^{2}}{3}-\frac{T_{f}}{C_{A}} \frac{59}{36}\right]
$$

b) for the subprocess $g+g \longrightarrow H+g$ :

$$
W_{g g}^{\mathrm{MC}}(\alpha, \beta)=\frac{1+z^{4}+\alpha^{4}+\beta^{4}}{1+z^{4}+(1-z)^{4}} \quad \leq 1,
$$

c) for the subprocess $g+q \longrightarrow H+q$ :

$$
W_{g q}^{\mathrm{MC}}(\alpha, \beta)=\frac{1+\beta^{2}}{1+(1-z)^{2}} \quad \leq 1
$$

In the above we use Sudakov variables defined as follows:

$$
\alpha=\frac{k p_{2}}{p_{1} p_{2}}, \quad \beta=\frac{k p_{1}}{p_{1} p_{2}}, \quad \alpha+\beta=1-z \leq 1, \quad \alpha \geq 0, \beta \geq 0 .
$$

They are visualized in Fig. 1. The immediate question is: which $\alpha, \beta-$ for which parton? The answer is simple: these of the parton with maximum $k_{T}$, i.e. for PS MC with the $k_{T}$-ordering, the ones of the 1st parton in the backward evolution. For the angular ordering, a parton with maximum $k_{T}$ needs to be found in the MC event.

The above NLO correcting weight is particularly simple because it is independent of the momenta of the Higgs-decay products, due to spin-zero of the Higgs boson. However, the NLO weight is also quite simple in the following case of gluonstrahlung in the $Z / \gamma$ production process:

$$
\begin{aligned}
d \sigma_{n}^{\mathrm{NLO}} & =\left(1+\Delta_{V S}+\sum_{i=1}^{n} W_{q \bar{q}}^{[1]}\left(\alpha_{i}, \beta_{i}\right)\right) d \sigma_{n}^{\mathrm{LO}} \\
W_{q \bar{q}}^{[1]} & =\frac{d^{5} \bar{\beta}_{q \bar{q}}}{d^{5} \sigma_{q \bar{q}}^{\mathrm{LO}}}=\frac{d^{5} \sigma_{q \bar{q}}^{\mathrm{NLO}}-d^{5} \sigma_{q \bar{q}}^{\mathrm{LO}}}{d^{5} \sigma_{q \bar{q}}^{\mathrm{LO}}}, \quad \Delta_{V S}^{q \bar{q}}=\frac{\alpha_{s}}{2 \pi} C_{F}\left[\frac{4}{3} \pi^{2}-\frac{5}{2}\right], \\
d^{5} \sigma_{q \bar{q}}^{N L O}(\alpha, \beta, \Omega) & =\frac{C_{F} \alpha_{s}}{\pi} \frac{d \alpha d \beta}{\alpha \beta} \frac{d \varphi}{2 \pi} d \Omega\left[\frac{d \sigma_{0}\left(\hat{s}, \theta_{F}\right)}{d \Omega} \frac{(1-\beta)^{2}}{2}+\frac{d \sigma_{0}\left(\hat{s}, \theta_{B}\right)}{d \Omega} \frac{(1-\alpha)^{2}}{2}\right], \\
d^{5} \sigma_{q \bar{q}}^{\mathrm{LO}}(\alpha, \beta, \Omega) & =d^{5} \sigma_{q \bar{q}}^{F}+d^{5} \sigma_{q \bar{q}}^{B}=\frac{C_{F} \alpha_{s}}{\pi} \frac{d \alpha d \beta}{\alpha \beta} \frac{d \varphi}{2 \pi} d \Omega \frac{1+(1-\alpha-\beta)^{2}}{2} \frac{d \sigma_{0}}{d \Omega}(\hat{s}, \hat{\theta}),
\end{aligned}
$$

The above formula is well-suited for PS MC with the angular ordering. Here, the MC weight sums up "democratically" contributions from all gluons, picking up automatically the contribution from the gluon with the maximum transverse momentum $k_{T}$. Alternatively, we may find out which gluon has maximum $k_{T}$ and reduce $\sum_{i=1}^{n}$ just to one term. In this way the KrkNLO scheme exploits the Sudakov suppression as in POWHEG, but there is no need of truncated or vetoed showers necessary in POWHEG for angular ordering.

In the case of averaging over the angles of $Z / \gamma$ decay products, the NLO weight is even simpler. For the subprocess $q+\bar{q} \longrightarrow Z$ it reads

$$
W_{V S}^{\mathrm{MC}}(\alpha, \beta)=1+\Delta_{V S}^{\mathrm{MC}}, \quad \Delta_{V S}^{\mathrm{MC}}=\frac{\alpha_{s}}{2 \pi}\left(\frac{4 \pi^{2}}{3}+\frac{1}{2}\right),
$$


for $q+\bar{q} \longrightarrow Z+g$ one gets

$$
W_{q \bar{q}}^{\mathrm{MC}}(\alpha, \beta)=\left\langle\frac{\left|\mathscr{M}_{q \bar{q} \rightarrow Z g}^{\mathrm{NLO}}\right|^{2}}{\left|\mathscr{M}_{q \bar{q} \rightarrow Z g}^{\mathrm{MC}}\right|^{2}}\right\rangle_{\mathrm{Z} \text { decay }}=1-\frac{2 \alpha \beta}{1+z^{2}} \leq 1,
$$

and for $q+g \longrightarrow Z+q$ one employs

$$
W_{q g}^{\mathrm{MC}}(\alpha, \beta)=\left\langle\frac{\left|\mathscr{M}_{q g \rightarrow Z q}^{\mathrm{NLO}}\right|^{2}}{\left|\mathscr{M}_{q g \rightarrow Z q}^{\mathrm{MC}}\right|^{2}}\right\rangle_{\mathrm{Z} \text { deday }}=1+\frac{\alpha(\alpha+2 z)}{z^{2}+(1-z)^{2}} \quad<3 .
$$

Obviously, the KrkNLO recipe is dramatically simpler than the POWHEG or MC@NLO methods. However, several non-trivial conditions have to be met before it can be applied. The most important requirements are:

- PDFs in the MC factorization scheme - the absolute must!

- LO PS MC must cover the entire NLO phase space.

- LO PS MC has to reproduce precisely all soft and collinear singularities of the NLO level a non-trivial requirement for $\mathrm{LO}$ processes with $\geq 3$ colored legs.

\section{Monte Carlo factorization scheme}

The LO PDFs in the MC factorization scheme in terms of the PDFs in the $\overline{M S}$ factorization scheme are defined by

$$
\left[\begin{array}{l}
q\left(x, Q^{2}\right) \\
\bar{q}\left(x, Q^{2}\right) \\
G\left(x, Q^{2}\right)
\end{array}\right]_{\mathrm{MC}}=\left[\begin{array}{c}
q \\
\bar{q} \\
G
\end{array}\right]_{\overline{M S}}+\int d z d y\left[\begin{array}{ccc}
K_{q q}^{\mathrm{MC}}(z) & 0 & K_{q G}^{\mathrm{MC}}(z) \\
0 & K_{\bar{q} \bar{q}}^{\mathrm{MC}}(z) & K_{\bar{q} G}^{\mathrm{MC}}(z) \\
K_{G q}^{\mathrm{MC}}(z) & K_{G \bar{q}}^{\mathrm{MC}}(z) & K_{G G}^{\mathrm{MC}}(z)
\end{array}\right]\left[\begin{array}{c}
q\left(y, Q^{2}\right) \\
\bar{q}\left(y, Q^{2}\right) \\
G\left(y, Q^{2}\right)
\end{array}\right]_{\overline{M S}} \delta(x-y z)
$$

where

$$
\begin{aligned}
& K_{G q}^{\mathrm{MC}}(z)= \frac{\alpha_{s}}{2 \pi} C_{F}\left\{\frac{1+(1-z)^{2}}{z} \ln \frac{(1-z)^{2}}{z}+z\right\}, \\
& K_{G G}^{\mathrm{MC}}(z)=\frac{\alpha_{s}}{2 \pi} C_{A}\left\{4\left[\frac{\ln (1-z)}{1-z}\right]_{+}+2\left[\frac{1}{z}-2+z(1-z)\right] \ln \frac{(1-z)^{2}}{z}-2 \frac{\ln z}{1-z}\right.\left.-\delta(1-z)\left(\frac{\pi^{2}}{3}+\frac{341}{72}-\frac{59}{36} \frac{T_{f}}{C_{A}}\right)\right\}, \\
& K_{q q}^{\mathrm{MC}}(z)=\frac{\alpha_{s}}{2 \pi} C_{F}\left\{4\left[\frac{\ln (1-z)}{1-z}\right]_{+}-(1+z) \ln \frac{(1-z)^{2}}{z}-2 \frac{\ln z}{1-z}+1-z-\delta(1-z)\left(\frac{\pi^{2}}{3}+\frac{17}{4}\right)\right\}, \\
& K_{q G}^{\mathrm{MC}}(z)=\frac{\alpha_{s}}{2 \pi} T_{R}\left\{\left[z^{2}+(1-z)^{2}\right] \ln \frac{(1-z)^{2}}{z}+2 z(1-z)\right\} .
\end{aligned}
$$

All virtual parts $\sim \delta(1-z)$ are adjusted using the PDFs momentum sum rules. 
Alternatively, direct fitting of the LO PDFs in the MC scheme directly to DIS data requires the following NLO coefficient functions of the DIS process in the MC scheme:

$$
\begin{aligned}
& C_{2, q q}^{\mathrm{MC}}(z)=\frac{\alpha_{s}}{2 \pi} C_{F}\left\{\left[-\frac{1+z^{2}}{1-z} \ln (1-z)-\frac{3}{2} \frac{1}{1-z}+3 z+2\right]_{+}+\frac{3}{2} \delta(1-z)\right\}, \\
& C_{2, q G}^{\mathrm{MC}}(z)=\frac{\alpha_{s}}{2 \pi} T_{R}\left\{-\left[z^{2}+(1-z)^{2}\right] \ln (1-z)+6 z(1-z)-1\right\},
\end{aligned}
$$

instead of the following ones of the $\overline{M S}$ scheme:

$$
\begin{aligned}
& C_{2, q q}^{\overline{M S}}(z)=\frac{\alpha_{s}}{2 \pi} C_{F}\left[\frac{1+z^{2}}{1-z} \ln \frac{1-z}{z}-\frac{3}{2} \frac{1}{1-z}+2 z+3\right]_{+}, \\
& C_{2, q G}^{\overline{M S}}(z)=\frac{\alpha_{s}}{2 \pi} T_{R}\left\{\left[z^{2}+(1-z)^{2}\right] \ln \frac{1-z}{z}+8 z(1-z)-1\right\} .
\end{aligned}
$$

Let us discuss briefly some important issues concerning the definition and the use of the MC factorization scheme. What is the main purpose of the MC factorization scheme? It is defined such that $\Sigma(z) \delta\left(k_{T}\right)$ terms due to emissions from initial partons disappear completely from the exclusive NLO corrections. Such terms exist in the fixed-order NLO calculations independently of matching with the PS MC ${ }^{1}$. It is vital to remove them in the KrkNLO scheme, because without eliminating them it is not possible to include the NLO corrections using simple multiplicative MC weights on top of the PS MC distributions.

How to determine elements of the transition matrix $K_{a b}^{\mathrm{MC}}$ ? They can be be obtained from inspecting NLO corrections to two processes with initial-state quarks and gluons in the LO hard process: $Z / \gamma$ and Higgs-boson production, as was done in ref. [13].

The immediate question, of course, is whether the same set of PDFs in the MC factorization scheme is able to eliminate unwanted $\sim \delta\left(k_{T}\right)$ terms for other processes? This is a question about universality of the MC factorization scheme. It was shown already in ref. [3], that this is true for the DIS process. Preliminary analysis shows that for any other process with any number of colored particles it may work, provided PS MC distributions (or the corresponding soft-collinear counter terms in the NLO calculations) obey certain extra conditions. The corresponding discussion will be reported/published in a separate study.

\subsection{On the quality of LO PS MC}

In the implementation of other methods of combining (matching) NLO-corrected hard process with LO PS, the MC generation of the distribution of the hardest parton responsible for the bulk of the LO+NLO corrections (POWHEG [10]) or missing NLO (MC@NLO [9]) is taken outside LO PS MC and is done using separate software tools, before LO PS MC is invoked to add more partons. In KrkNLO the order is inverse, PS MC is invoked first to generate the hardest parton and other partons together and then the NLO corrections are introduced in the next step by means of reweighting the generated $\mathrm{MC}$ events.

Assuming for simplicity the $k_{T}$-ordering in PS MC, the distribution of the hardest parton generated by LO PS MC has to be controlled quite strictly, that is analytically ${ }^{2}$. It has also to reproduce

\footnotetext{
${ }^{1}$ For instance in ref. [14] they are termed as "collinear remnants".

${ }^{2}$ This is true for other methods as well.
} 
properly soft and collinear limits of the NLO distributions. For simple processes with two initialstate hadrons and any color-neutral object in the final state, like DY or Higgs-boson production, the above requirements are easily fulfilled by any modern PS MCs. In ref. [4] which implements the KrkNLO method for the DY process it was checked very carefully that the LO distributions of Sherpa 2.0.0 and Herwig++ 2.7.0 are identical with the soft-collinear counter terms of the Catani-Seymour subtraction scheme [14].

Another related critical point is the use of the backward evolution (BEV) algorithm in LO PS MC. It is easy to prove that the LO backward and forward evolutions are the same up to LO. However, the control of the NLO terms in the BEV algorithm is less trivial, especially for the terms due to kinematic limits. This problem was analyzed in a detail in ref. [4], where it was shown that such a strict NLO-level control of the distributions generated by BEV algorithm is possible. In particular, the role of the veto algorithm in BEV in assuring the full coverage of the phase space up to $k_{T}=\sqrt{s} / 2$ was clarified.

In the case of more than three colored partons in the LO hard process, the requirement on PS MC that it reproduces exactly the QCD soft-collinear limit is difficult to meet and typical LO PS MCs implement some approximate distributions, which have to be corrected once the NLO corrections are implemented using the KrkNLO method (provided the full coverage of the soft gluon phase space is assured).

\subsection{Numerical examples of PDFs in the MC factorization scheme}

In Fig. 2 we show a few examples of PDFs transformed from the $\overline{M S}$ to the MC factorization scheme. They will be used in the following section for the Higgs-production process.

As seen in Fig. 2, the change of PDFs in the MC scheme with respect to the $\overline{M S}$ is noticeable. Lines labeled with $\mathrm{MC}$ represent the complete MC scheme as defined in eq. (3.1). Lines marked with $\mathrm{MC}_{D Y}$ use simplified $K$-matrix of ref. [4]. This simplification was acceptable for the DY process modulo $\mathscr{O}\left(\alpha_{s}^{2}\right)$ terms. Generally, for low $x$ the gluon PDF is smaller for the $\overline{M S}$ than for the MC scheme, while for quarks it is the other way around.

\section{Higgs-production process}

The application of the KrkNLO method for the DY process was covered in a great detail in ref. [4], where numerical results for the total cross section, distributions of rapidity and transverse momentum were shown for the KrkNLO method, in comparison with fixed-order NLO of MCFM [11], NNLO of ref. [12] and also with the results of the POWHEG [10] and MC@NLO [9] implementations.

Similar extensive panorama of numerical results for the Higgs-production process using the KrkNLO method will be published soon [15]. Here we are going to show preliminary results for an integrated cross section. In the table below we include the results for the total cross section of the Higgs production in pp collisions at $\sqrt{s}=8 \mathrm{TeV}$ from the KrkNLO method compared with that of MC@NLO [9]:

\begin{tabular}{l|l} 
& $\sigma_{t o t}[p b]$ \\
\hline MC@NLO & $18.72 \pm 0.04$ \\
KrkNLO & $19.38 \pm 0.04$
\end{tabular}



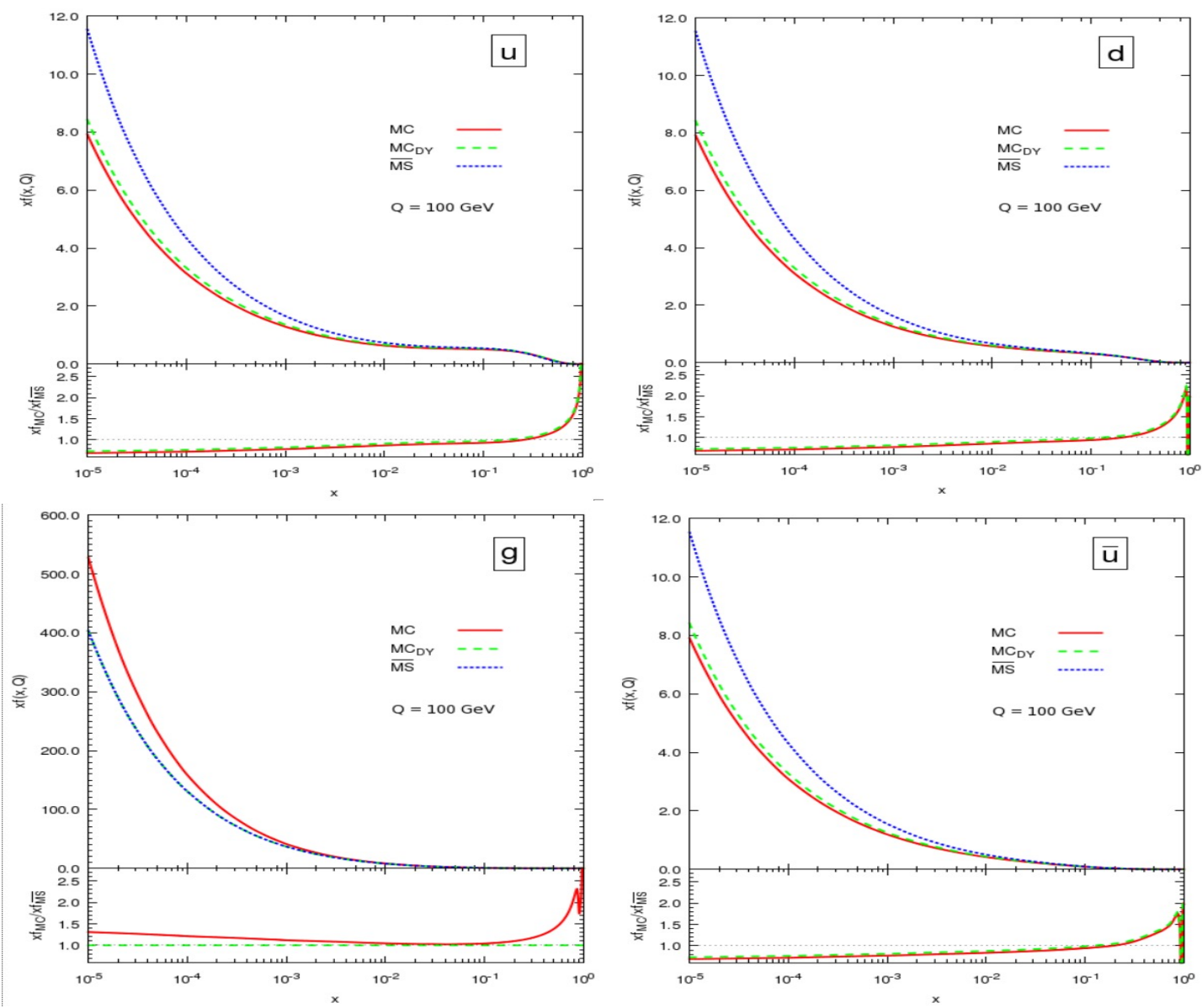

Figure 2: Examples of PDFs in the MC factorization scheme.

The difference of $\sim 3.5 \%$ can be attributed to different treatment in the two matching methods of effects that are beyond the strict NLO approximation.

In the preliminary results, to be shown in ref. [15], the rapidity distributions from the KrkNLO method implemented on top of the most recent version of Herwig 7.0 [16], differs by $\leq 20 \%$ from that of MC@NLO [9] methods, well within uncertainty typical for NLO. A similar difference, typical for NLO, was found in transverse momentum distribution for $p_{T} \leq 100 \mathrm{GeV}$. For higher transverse momenta 200\% differences with MC@NLO are found, well known from comparisons between POWHEG and MC@NLO for this process, see ref. [17].

\section{Summary and outlook}

The KrkNLO method of introducing the NLO QCD corrections in the LO parton shower Monte Carlo is a simple scenario for the NLO-corrected PS MC - an interesting alternative to the more complicated MC@NLO or POWHEG approaches. Potential gains from the new QCD matching method are: (i) reducing h.o. QCD uncertainties, (ii) possibly easier implementation of NNLO corrections. An interesting application would be a high-quality QCD+EW+QED Monte Carlo event generator with a hard process like single or multiple $W / Z / H$ heavy boson production at 
high luminosity LHC. A longer-term possibility is to extend the KrkNLO technique to the N+NLO calculation, with the NLO ladder (PS MC) and NNLO-corrected hard process.

\section{References}

[1] M. Skrzypek et al., Acta Phys.Polon. B42, 2433 (2011), 1111.5368.

[2] S. Jadach, M. Jezabek, A. Kusina, W. Płaczek, and M. Skrzypek, Acta Phys.Polon. B43, 2067 (2012), 1209.4291.

[3] S. Jadach, A. Kusina, W. Płaczek, M. Skrzypek, and M. Slawinska, Phys.Rev. D87, 034029 (2013), 1103.5015 .

[4] S. Jadach, W. Płaczek, S. Sapeta, A. Siódmok, and M. Skrzypek, JHEP 10, 052 (2015), 1503.06849.

[5] T. Gleisberg et al., JHEP 02, 007 (2009), 0811.4622.

[6] M. Bahr et al., Eur.Phys.J. C58, 639 (2008), 0803.0883.

[7] J. Bellm et al., (2013), 1310.6877.

[8] S. Gieseke, C. Rohr, and A. Siodmok, Eur.Phys.J. C72, 2225 (2012), 1206.0041.

[9] S. Frixione and B. R. Webber, JHEP 06, 029 (2002), hep-ph/0204244.

[10] P. Nason, JHEP 11, 040 (2004), hep-ph/0409146.

[11] J. M. Campbell and R. K. Ellis, Phys.Rev. D60, 113006 (1999), hep-ph/9905386, http://mcfm.fnal.gov.

[12] S. Catani, L. Cieri, G. Ferrera, D. de Florian, and M. Grazzini, Phys. Rev. Lett. 103, 082001 (2009), 0903.2120.

[13] S. Jadach, W. Płaczek, S. Sapeta, A. Siódmok, and M. Skrzypek, (2016), 1606.00355.

[14] S. Catani and M. H. Seymour, Nucl. Phys. B485, 291 (1997), hep-ph/9605323.

[15] S. Jadach et al., 2016, IFJPAN-IV-2016 report, in preperation.

[16] J. Bellm et al., Eur. Phys. J. C76, 196 (2016), 1512.01178.

[17] S. Alioli, P. Nason, C. Oleari, and E. Re, JHEP 04, 002 (2009), 0812.0578. 